\title{
Metastatic Alveolar Soft Part Sarcoma
}

National Cancer Institute

\section{Source}

National Cancer Institute. Metastatic Alveolar Soft Part Sarcoma. NCI Thesaurus. Code C8796.

An alveolar soft part sarcoma which has spread to another anatomic site. 